\section{Mapping of QTL for aluminum tolerance in tropical maize}

\author{
Caroline de Jesus Coelho ${ }^{1}$, José Raulindo Gardingo ${ }^{1}$, Mara \\ Cristina de Almeida ${ }^{1}$ and Rodrigo Rodrigues Matiello ${ }^{1^{*}}$
}

\begin{abstract}
The objectives of this study were to map genomic regions associated with QTL for aluminum (Al) tolerance in maize and determine the phenotypic effects of tolerance loci. QTL analysis for Al tolerance was carried out in a population of $F_{2: 3}$ progenies resulting from a cross between the contrasting lines $L_{T} 99-4$ and $L_{s}$ 04-2. SSR (Simple Sequence Repeat) loci and AFLP (Amplified Fragment Length Polymorphism) were used to construct the linkage map and to detect QTL mapped by composite interval mapping analysis. Nine tolerance QTL among eight linkage groups (chromosomes 2, 4, 5, 6, 7, 8, 9, and 10) were mapped, which explained $70.3 \%$ of the phenotypic variance. The results confirmed three major QTL (bins 6.00, 8.05, and 10.01) that are described in the literature for Al tolerance, which accounted for most of the phenotypic variance (40.3\%).
\end{abstract}

Keywords: Tolerance loci, composite interval mapping, molecular markers, linkage map.

\section{INTRODUCTION}

Aluminum (Al) toxicity has been recognized as the factor that most limits crop production in acid soils. Studies indicate that acid soils constitute approximately $50 \%$ of cultivated soils in the world. Al toxicity reduces growth of the plant root system, making crops more susceptible to water stress and mineral deficiency, which reduces yield. The toxic effect of Al can strongly affect the maize crop, causing yield reductions of more than $80 \%$ (Doncheva et al. 2005).

In recent decades, great efforts have been made to understand the mechanisms responsible for Al tolerance in plants. Al tolerant genotypes have been identified in different plant species; however, the physiological mechanisms and genetic factors responsible for Al tolerance are still poorly understood. Genetic studies suggest that Al tolerance in maize is a complex characteristic (Pandey et al. 2007), probably involving multiple genes and, consequently, several physiological mechanisms (Sibov et al. 1999, Ninamango-Cárdenas et al. 2003, Piñeros et al. 2005).

Using root growth as the phenotypic index, QTL mapping studies for Al tolerance resulted in identification of genomic regions associated with Al tolerance on maize chromosomes 1, 2, 4, 5, 6, 8, and 10 (Sibov et al. 1999, NinamangoCárdenas et al. 2003, Conceição et al. 2009, Krill et al. 2010). However, only a few of these regions coincided among the studies performed, due to the low saturation of molecular marker linkage maps and the large physical distances (cM) between them.
Crop Breeding and Applied Biotechnology 19: 86-94, 2019 Brazilian Society of Plant Breeding. Printed in Brazil http://dx.doi.org/10.1590/198470332019v19n1a12 \title{
(1)
}


Combining linkage analysis and associative mapping, Krill et al. (2010) chose four gene candidates possibly associated with Al tolerance in temperate maize. The genes ZmASL (Zea mays AltSB like), ZmALMT2 (Zea mays aluminum-activated malate transporter2), SAHH (S-adenosyl-L-homocysteinase) and ME (Malic Enzyme) explained from 13 to $20 \%$ of phenotypic variance of net root growth. In another study, Maron et al. (2010) identified and characterized two members of the MATE family (Multidrug and Toxic Compound Extrusion), ZmMATE1 and ZmMATE2, as major genes controlling Al tolerance in temperate maize. The gene ZmMATE1, mapped on the telomeric region of chromosome 6, codes a transmembrane protein that mediates citrate exudation on the maize root apex. This genetic result confirms physiological studies that point to root citrate exudation as the main Al tolerance mechanism in temperate maize (Piñeros et al. 2002). However, ZmMATE2 characterization (mapped at chromosome 5) showed that this gene is different from ZmMATE1 and the other MATEs involved in Al tolerance in many aspects. According to Maron et al. (2010), ZmMATE2 and MATEs do not share the same identity sequence indicated for citrate transport, being involved with a different tolerance mechanism. However, Piñeros et al. (2005) suggest the existence of other complementary mechanisms for Al tolerance in this species. Recently, Guimarães et al. (2014) identified the gene ZmNrat1, homologous to the OsNrat1 gene that codes a specific Al transporter involved in Al tolerance in rice. This region was mapped on chromosome 5 of maize in the proximity of the ZmMATE2 gene.

QTL mapping is reportedly one of the most efficient ways of associating phenotypic characteristics to genetics, which contributes significantly to genetic breeding programs. Thus, the objectives of this study were to identify genomic regions associated with Al tolerance QTL and quantify the phenotypic effects of these QTL with tolerance to this element in tropical maize germplasm.

\section{MATERIAL AND METHODS}

\section{Phenotypic evaluation of $F_{2: 3}$ progenies}

From crossing inbred lines L 04-2 (sensitive) and L 99-4 (tolerant), the $F_{1}$ and $F_{2}$ generations were obtained. Individuals of the $F_{2}$ generation were self-pollinated, giving rise to the $F_{2: 3}$ progenies, which were phenotypically evaluated for $\mathrm{Al}$ tolerance in minimal solution. The treatments consisted of $350 \mathrm{~F}_{2: 3}$ maize progenies, as well as the parents $\left(\mathrm{L}_{\mathrm{T}} 99-4\right.$ and $L_{\mathrm{s}}$ 04-2) and $F_{1}$ generation, which were used as experimental controls. The experiment used a randomized block design with three replications, and 12 seedlings of each progeny were evaluated per replication. After seed germination, the initial length (IL) of the main root was measured. After that, the seedlings were placed in expanded polystyrene trays and then deposited in a tank containing of solution of $4 \mathrm{mg} \mathrm{L}^{-1} \mathrm{Al}+40 \mathrm{mg} \mathrm{L}^{-1} \mathrm{Ca}$. After 48 hours of exposure, the length of the main root was measured again (FL - final length). The difference between IL and FL (FL $-\mathrm{IL}$ ) in cm was calculated (called DIF) (Coelho et al. 2015).

The DIF averages of $F_{2: 3}$ data were subjected to statistical analysis of variance by $R$ software ( $R$ Core Team 2017). The number of phenotypic classes $(k)$ was estimated by $k=\sqrt{n}$, where $n$ refers to the total number of progenies $(n=350)$. The phenotypic amplitude of each class (PAC) was determined by PAC $=\frac{A}{K}$, where A represents the DIF amplitude observed between the maize progenies.

\section{Genotyping of $F_{2}$ plants}

Genomic DNA was extracted from individual plant leaves of the $F_{2}$ generation, inbred lines $\left(L_{T} 99-4\right.$ and $\left.L_{S} 04-2\right)$, and $F_{1}$ generation. The extraction of genomic DNA followed the Hoisington et al. (1994) protocol. The DNA was resuspended in $40 \mu \mathrm{L}$ sterile ultrapure water and treated with $2 \mu \mathrm{L}$ of RNase $\left(1.0 \mathrm{mg} \mathrm{mL}^{-1}\right)$ for 2 hours at $37^{\circ} \mathrm{C}$ in a water-bath.

Seventy-nine (79) microsatellite loci (SSR) distributed in 10 linkage groups of the maize consensus map were amplified from the inbred lines ( $L_{T} 99-4$ and $L_{S}$ 04-2) in order to select polymorphic loci between parental lines. The SSR loci were selected from the Maize Genetics and Genomics Data Base - MaizeGDB (http://www.maizegdb.org). The amplification reactions were conducted in the Biocycler Biosystems ${ }^{\circledR}$ thermocycler based on Senior et al. (1996) and Ogliari et al. (2000) protocols. The polymerase chain reaction (PCR) was prepared in $0.2 \mathrm{~mL}$ micro tubes, containing $1 \mathrm{X}$ buffer (200 $\mathrm{mM}$ Tris- $\mathrm{HCl}$ - pH 8.4, $500 \mathrm{mM} \mathrm{KCl}$, Invitrogen); $2.0 \mathrm{mM} \mathrm{MgCl} ; 0.1 \mathrm{mM}$ of dNTP solution (100 mM, Amresco $\left.{ }^{\circledR}\right) ; 0.2 \mu \mathrm{M}$ of each primer (forward and reverse); $0.75 \mathrm{U}$ of Taq DNA polymerase (5 $\mathrm{U} \mu \mathrm{L}^{-1}$, Invitrogen); and $40 \mathrm{ng}$ of DNA for a final volume of $20 \mu \mathrm{L}$. For optimization of different SSR loci, three PCR programs were used: two touchdown types (Ogliari et 
al. 2000) and one of normal cycling. The amplified samples were stained with $4 \mu \mathrm{L}$ of GelRed $0.1 \mathrm{X}$ plus loading buffer $(1: 1)$ and separated by electrophoresis in 2\% agarose gel in TBE $1 X$ Buffer for about 3 hours at $80 \mathrm{~V}$. The 350 individuals $\left(F_{2}\right)$ of the mapping population were genotyped for each polymorphic locus with the following codification: $(-1)$ amplified fragment corresponding to the SSR allele from sensitive line $L_{s}$ 04-2, (1) amplified fragment corresponding to the SSR allele from tolerant line $L_{T} 99-4$, and (0) presence of two fragments corresponding to alleles from both parental lines, $\mathrm{L}_{\mathrm{S}}$ 04-2 and $\mathrm{L}_{\mathrm{T}}$ 99-4.

Initially, 25 combinations of EcoRI and Msel primers were tested in order to select those combinations with better amplification patterns and greater polymorphism between the parental lines. Using this criterion, 12 combinations were chosen for genotyping the mapping population. The amplification methodology of the AFLP (Amplified Fragment Length Polymorphism) marker was performed according to Vos et al. (1995). The selective amplification products were analyzed by polyacrylamide (6\%) gel electrophorese at $80 \mathrm{~W}$ potency for approximately 3 hours. The gels were silver stained according to Creste et al. (2001). The polymorphic amplified fragments for each EcoRI + Msel primer combination in the mapping population were genotyped for the presence (1) or absence (0) of the fragment.

\section{Linkage analysis and composite interval mapping}

The SSR and AFLP loci, which showed expected Mendelian segregation (1:2:1 and 1:1 for the SSR and AFLP markers, respectively) by chi-square at $5 \%$, were used for linkage map construction by the MAPMAKER 3.0 program (Lander et al. 1987). Linkage group construction was based on the "group" command using an LOD of 3.0 (maximum likelihood method) and maximum recombination frequency of $\theta=0.20$. The recombination frequencies were converted into map distances by the Haldane's Mapping Function (2008). After the linkage groups were defined, the markers inside each group were ordered by the "order" command with LOD higher than 3.0. To ensure correct marker order, the "ripple" command was used.

The QTL mapping analysis was made using the DIF averages of $350 \mathrm{~F}_{2: 3}$ progenies from the cross between $\mathrm{L}_{\mathrm{T}} 99-4$ $x L_{s}$ 04-2 evaluated for Al tolerance. Composite interval mapping analysis (CIM) was used for QTL mapping with the QTLCartographer program (Basten et al. 2005). CIM linkage map analysis of molecular markers generated by MAPMAKER 3.0 was used for QTL positioning. The estimated position, genetic effect, and percent of phenotypic variance (given by the partial correlation coefficient, $\mathrm{R}^{2}$ ) of QTL was established at the LOD peak above the level considered significant (3.0) according to the QTLCartographer ${ }^{\circledR}$ program.

\section{RESULTS AND DISCUSSION}

\section{Phenotyping of $\mathrm{F}_{2: 3}$ progenies}

The results of analysis of variance showed a significant effect $(p<0.01)$ among the treatments $\left(F_{2: 3}\right.$ progenies, parental lines, and $F_{1}$ generation) for the DIF variable. The coefficient of variation had a lower level (6.9\%), appropriate for phenotyping experiments (data not shown). The decomposition of phenotypic variance components indicated predominance of genetic variance in relation to environmental variance. The broad sense heritability coefficient was estimated and it showed large magnitude (0.99).

The frequency distribution of maize $F_{2: 3}$ progenies in the DIF classes is shown in Figure 1. A tendency of symmetric distribution among the DIF phenotypic classes was identified, similar to a normal distribution curve, with classes ranging from 0.75 to $2.62 \mathrm{~cm}$. Analysis confirmed the phenotypic contrast for Al tolerance between parental lines $L_{T} 99-4$ (tolerant) and $L_{S}$ 04-2 (sensitive) through difference in

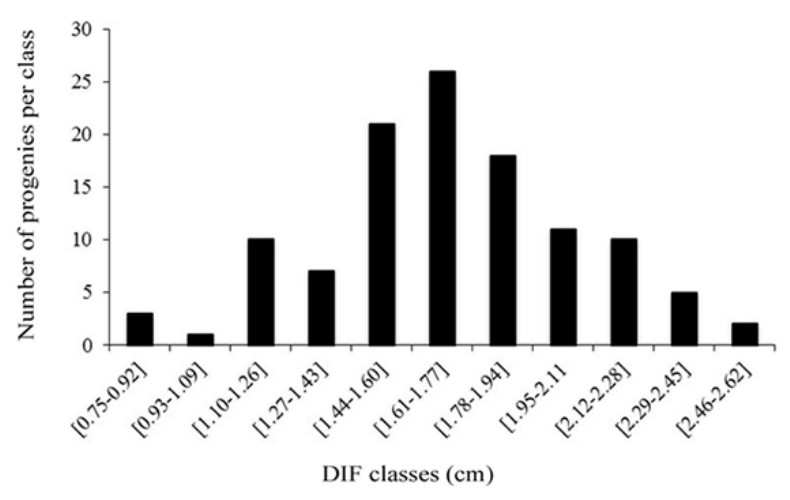

Figure 1. Distribution of $\mathrm{F}_{2: 3}$ maize progenies originated by the cross $L_{\text {S }}$ 99-4 $\times L_{S}$ 04-2 on DIF (differential root growth) classes. $\mathrm{L}_{\mathrm{T}}=$ tolerant inbred line, $\mathrm{L}_{\mathrm{S}}=$ sensitive inbred line, and $\mathrm{F}_{1}=\mathrm{F}_{1}$ generation. 
root growth. The tolerant line $L_{T} 99-4$ was in the phenotypic class of 1.27 to $1.43 \mathrm{~cm}$, along with the $F_{1}$ generation. In contrast, the sensitive line $L_{s}$ 04-2 ranked in the 1 st phenotypic class $(0.75$ to $0.92 \mathrm{~cm})$. Only four progenies $(3.5 \%)$ were ranked in sensitive classes (up to $1.09 \mathrm{~cm}$ ). Furthermore, transgressive segregants were observed in this population, since $82 \%$ (286) of the progenies were above $1.43 \mathrm{~cm}$, which was higher than the tolerant parental line $\mathrm{L}_{\mathrm{T}} 99-4$ and the $\mathrm{F}_{1}$ generation (Figure 1).

The high amplitude of DIF $(0.75$ to $2.62 \mathrm{~cm})$ of the $\mathrm{F}_{2: 3}$ progenies evaluated showed higher genetic variability for Al tolerance. This may be due to hypothesized multiple tolerance mechanisms acting in maize Al tolerance. Piñeros et al. (2005) described that, in addition to citrate exudation by roots, there are other physiological mechanisms, such as Al complexation in the cellular vacuoles and rhizosphere $\mathrm{pH}$ modification, that may be involved.

\section{Linkage map}

Of the 444 polymorphic fragments (SSR and AFLP), only 254, with expected Mendelian segregation (not distorted), were used in linkage map construction. Two hundred and forty-six (246) markers were grouped between the 10 maize linkage groups (chromosomes) using 3.0 LOD as grouping criterion, $\theta=0.20$ as maximum recombination frequency, and the Haldane's Mapping Function. As the SSR loci have known genomic location, correspondence of obtained linkage groups was established with 10 maize chromosomes (Figure 2). The chromosomal localization of the AFLP fragments was obtained by the results of linkage analysis with microsatellite loci, which have known location in the consensus maize map (Senior et al. 1996). The SSR loci thus acted as "anchors" for AFLP fragments without previously known locations. Thus, it is possible to develop linkage maps with high saturation since the cost of AFLP is lower, and the number of loci sampled per reaction for this marker is higher than for the microsatellite markers. Of the grouped markers, 218 (88\%) were ordered in 10 linkage groups, using LOD > 3.0. The number of ordered markers ranged from 18 in chromosome 7 to 31 in chromosome 8, with an average of 21.8 markers per chromosome. Total length was $1897.0 \mathrm{cM}$ in the linkage map, with average distance between markers of $8.8 \mathrm{cM}$. The length of the linkage groups ranged from $116.7 \mathrm{cM}$ in group 7 to $244.8 \mathrm{cM}$ in group 4 (Figure 2).

\section{QTL mapping}

Composite interval mapping (CIM) enabled mapping of nine QTL in different genomic regions associated with $\mathrm{Al}$ tolerance in the maize germplasm. QTL were detected in nine intervals distributed among 8 chromosomes $(2,4,5,6,7$, 8,9 , and 10). The phenotypic variance proportion shown by these QTL ranged from 2.1\% for the QTL on chromosome 2 at position $34.0 \mathrm{cM}$ to $17.7 \%$ for the QTL on chromosome $8(149.0 \mathrm{cM})$, which had the highest LOD score $(20.8)$ at the E44M5187 - Bnlg1176 interval. Together, these nine QTL explain 70.3\% of phenotypic variance for the DIF variable. The additive DIF effects ranged from 0.56 for the QTL at position $125.0 \mathrm{cM}$ of chromosome 4 to 0.55 for the QTL mapped at position $149.0 \mathrm{cM}$ of chromosome 8 (Table 1 ).

In spite of the large number of mapped QTL, there is no other map study that displayed wide QTL distribution over the maize genome located at chromosomes 2, 4, 5, 6, 7, 8, 9, and 10. Sibov et al. (1999) mapped 2 genomic regions associated with Al tolerance QTL ( $A / m 1$ and $A / m 2$ at chromosomes 10 and 6 , respectively) from a $F_{2}$ population obtained by crosses between lines that contrast regarding Al tolerance. The authors describe that the $A / m 1$ gene contributes most to phenotypic variance in net root growth (24.2\%), which was three times greater than Alm2 (7.7\%). Ninamango-Cárdenas et al. (2003) mapped five genomic regions involved with Al tolerance. Here the QTL were located at chromosomes 2, 6, and 8 using $\mathrm{CIM}$, and they explained $60 \%$ in phenotypic variance of tolerance. Similarly, Conceição et al. (2009) identified five genomic regions associated with Al tolerance QTL at chromosomes 4, 5, 6, 8, and 10. Those QTL explained a total of $41 \%$ of phenotypic variance, with the biggest association of root growth obtained by QTL at chromosome 10, which was responsible for $15 \%$ of phenotypic variance in Al tolerance.

From genomic and physiologic approaches, Krill et al. (2010) selected 22 candidate genes for Al tolerance, which were evaluated by association analysis. Of those studied genes, six presented significant results for tolerance expression by association analysis. However, only four were confirmed by linkage analysis. The candidate genes ZmASL, ALMT2, SAHH and $M E$, were mapped on chromosomes 1, 10,6, and 4, respectively, being responsible for $13 \%$ to $20 \%$ of phenotypic variance in net root growth. Recently, Guimarães et al. (2014) have identified five genomic regions significantly associated to Al tolerance QTL, by using 54,455 SNP markers (Single Nucleotide Polymorphism) in a RIL population derived from 
Cateto line Al237. In the mapped regions on chromosomes 2, 3, 5, 6, and 8 accounted for $62.8 \%$ of phenotypic variance in tolerance.
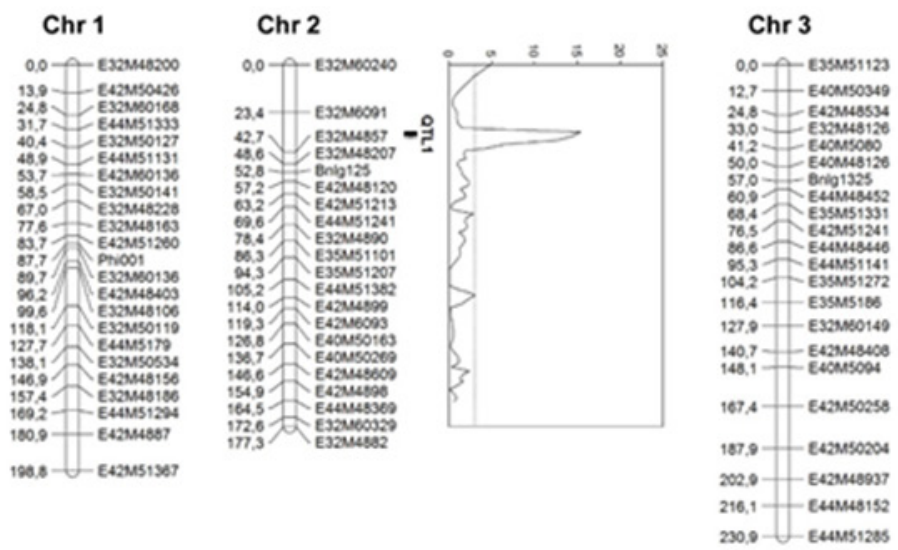

\section{Chr 5}

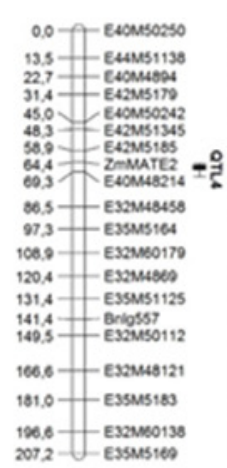

Chr 8

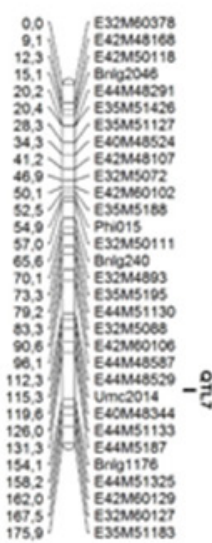

Chr 6

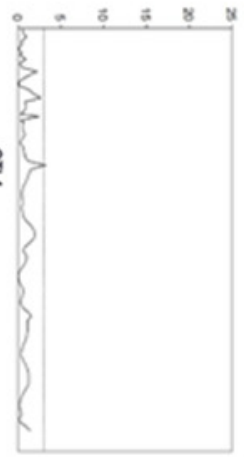

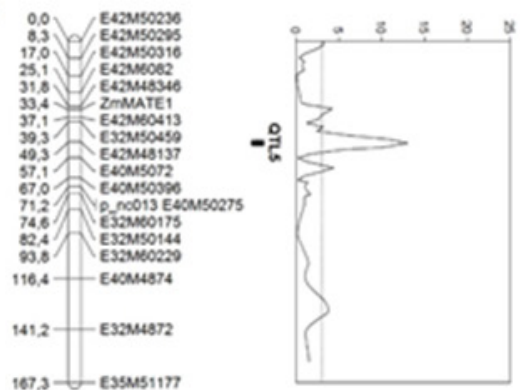

Chr 7

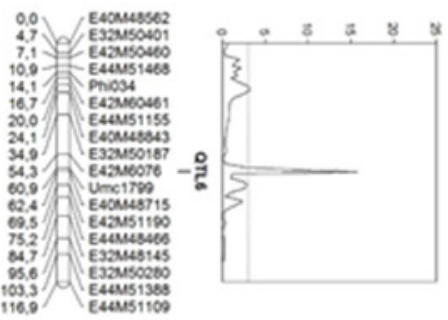

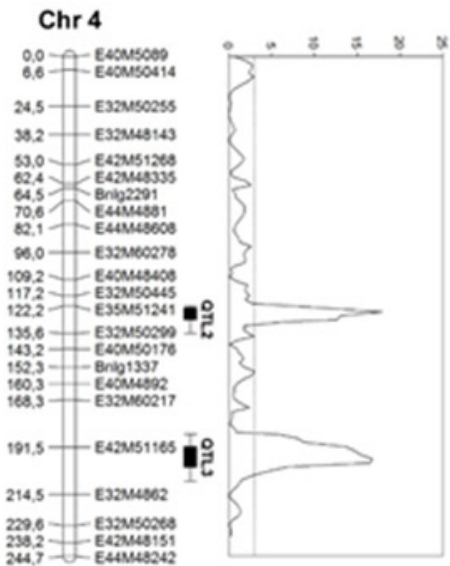


Table 1. LOD score, position (cM), proportion of phenotypic variance $\left(R^{2}\right)$, and estimate of additive (a) and dominance (d) effect explained by QTL mapped by the composite interval mapping methodology in a maize $\mathrm{F}_{2: 3}$ population

\begin{tabular}{|c|c|c|c|c|c|c|c|}
\hline \multirow{2}{*}{$\begin{array}{l}\text { Chr }^{\mathrm{a}} \\
2\end{array}$} & \multicolumn{2}{|c|}{ Marker interval } & \multirow{2}{*}{$\frac{\text { LOD }}{15.4}$} & \multirow{2}{*}{$\begin{array}{c}\text { Position (cM) } \\
34.0\end{array}$} & \multirow{2}{*}{$\frac{\mathbf{R}^{2}(\%)^{\mathbf{b}}}{2.11}$} & \multirow{2}{*}{$\frac{a^{c}}{-0.52}$} & \multirow{2}{*}{$\frac{d^{d}}{-0.54}$} \\
\hline & E32M6091 & E32M48207 & & & & & \\
\hline & 23.4 & 42.7 & & & & & \\
\hline \multirow[t]{2}{*}{4} & E35M51241 & E32M50299 & 17.9 & 125.0 & 5.57 & -0.56 & -0.46 \\
\hline & 122.2 & 135.6 & & & & & \\
\hline \multirow[t]{2}{*}{4} & E42M51165 & E32M4862 & 16.7 & 197.0 & 8.70 & -0.51 & -0.56 \\
\hline & 185.0 & 208.0 & & & & & \\
\hline \multirow[t]{2}{*}{6} & E42M48137 & E40M5072 & 13.0 & 51.0 & 10.57 & 0.01 & 1.05 \\
\hline & 49.3 & 57.1 & & & & & \\
\hline \multirow[t]{2}{*}{7} & E40M48715 & E42M51190 & 15.7 & 63.0 & 2.71 & 0.03 & 0.91 \\
\hline & 62.4 & 69.5 & & & & & \\
\hline 8 & E44M5187 & Bnlg1176 & 20.8 & 149.0 & 17.68 & 0.55 & -0.47 \\
\hline \multirow{2}{*}{10} & 158.5 & 181.7 & & & & & \\
\hline & $R_{T}^{2} e^{2}$ & & & & 70.3 & & \\
\hline
\end{tabular}

${ }^{a} \mathrm{Chr}=$ Chromosome; ${ }^{b} \mathrm{R}^{2}$ : proportion of phenotypic variance explained by $\mathrm{QTL}$ at the position considering $\mathrm{H} 3(\mathrm{a} \neq 0, \mathrm{~d} \neq 0)$ in relation to $\mathrm{HO}(\mathrm{a}=0, \mathrm{~d}=0) ;{ }^{\mathrm{c}} \mathrm{a}$ : estimate of additive effect of QTL under H3; ${ }^{d}$ d: estimate of dominance deviation effect under $\mathrm{H} 3$; ${ }^{\text {e }} \mathrm{R}_{\mathrm{T}}{ }_{\mathrm{T}}=$ proportion of total phenotypic variance explained by QTL

In general, many studies of QTL mapping associated with Al tolerance in maize have indicated chromosome 6 as having the highest number of identified QTL (Sibov et al. 1999, Ninamango-Cárdenas et al. 2003, Conceição et al. 2009, Krill et al. 2010, Guimarães et al. 2014). In our study, QTL were mapped at the $51 \mathrm{cM}$ position of chromosome 6 , only $17.6 \mathrm{cM}$ from marker ZmMATE1, which refers to a mapped gene in the telomeric region of chromosome 6 (bin 6.00) by Maron et al. (2010).

Ninamago-Cárdenas et al. (2003) located QTL2 at the end of chromosome 6 (bin 6.00), which was first identified by the BSA methodology and then confirmed by linear regression analysis and composite interval mapping. The authors associated this QTL with those that were described by Sibov et al. (1999) and identified as Alm2, confirming that this region may anchor genes involved in Al tolerance. Maron et al. (2010) attribute this region to the gene ZmMATE1 that encodes a transmembrane protein that mediates citrate efflux on the maize root apex. Al exclusion from the root apex by citrate exudation is largely recognized as the main mechanism of Al tolerance in the maize crop (Piñeros et al. 2005). In contrast with this assertion, Matiello et al. (2012) reported another mechanism that may be associated with Al tolerance in maize, through analysis of associations of microarray data described for Al tolerance QTL. Others authors mapped a constitutive gene in the same region of chromosome 6 that is highly identified with the gene SMP1 (Swellmap 1 gene). In studies of organogenesis and cell growth, Clay and Nelson (2005) found that one mutant genotype of Arabdopsis had knockout for this gene, resulting in smaller leaves and number of cells. This phenotype is caused by an early halt in cell division in leaf primordia; however, overexpression of this gene reduced leaf size, but increased the final number of cells. As observed by Doncheva et al. (2005), Al interferes with the cell cycle and with differentiation of the cells of the root apex in maize. Therefore, constitutive expression of the SMP1 gene in a tolerant genotype can maintain cell division even when its roots are subjected to stress. More recently, Guimarães et al. (2014) confirmed the presence of a QTL (qALT6) associated with the gene ZmMATE1. The authors showed that high expression of this gene doubles the tolerance in maize NILS (Near-Isogenic Lines) when qALT6 is introgressed in sensitive lines. These NILS exhibited three copies of the ZMMATE1 gene, confirming that the tolerance allele had been transferred. These studies corroborate research by Maron et al. (2010) in which the ZmMATE1 adjacent to the qALT6 region increased Al tolerance, and it was regulated by the presence of abiotic stress (Al).

In addition to the bin 6.00 genomic region, Ninamango-Cárdenas et al. (2003) mapped a QTL at bin 6.05 for the 
same chromosome, explaining $15.6 \%$ of phenotypic variance in root growth. Conceição et al. (2009) have recently mapped the same region. From linkage analysis and associated mapping combination, Krill et al. (2010) associated that region with the $M E$ (malic enzyme) gene, which catalyzes the conversion of malate to pyruvate. Thus, this gene can help regulate malate concentration in the cytosol, which was described as the main mechanism of Al tolerance in wheat. In this regard, malate, associated with another organic acid exudation, would act to chelate the Al absorbed via the apoplast and detoxify the root apex.

In this study, the higher percentage of DIF phenotypic variance (17.7\%) was explained by QTL mapped on chromosome 8 , associated with microsatellite marker Bnlg1176 and located at bin 8.05. Similar results were found by NinamangoCárdenas et al. (2003) who mapped a QTL associated with Al tolerance next to this bin (8.04). The same region has recently been confirmed by mapping studies developed by Conceição et al. (2009) and Guimarães et al. (2014). The authors associated this region with the $i d h /$ gene, which codes for isocitrate dehydrogenase, an enzyme related to the tricarboxylic (citric) acid cycle and other reactions that involve organic acid metabolism. In addition, Matiello et al. (2012) have identified a QTL at the region described by Ninamango-Cárdenas et al. (2003). However, these authors associated this region with the gene that codes proteins like Pirin. In studies with tomato cells in a suspension with an inducer of cell death, Orzaez et al. (2001) verified significant expression of the LePirin gene. This gene is an ortholog to the PIRIN protein present in humans that is involved in transcription of the NE-kB factor, which promotes transcription of several anti-apoptosis genes. In studies with two contrasting inbred lines tolerant to Al, Boscolo et al. (2003) demonstrated that root apex cells from a sensitive line died after Al exposure. In contrast, the tolerant line did not exhibit this response. These results indicate that proteins like Pirin are involved in activation of anti-apoptosis genes, which extend cell viability during Al exposure.

The QTL mapped on chromosome 10, 21 cM from the SSR marker Umc1319 (bin 10.01), also made a considerable contribution to phenotypic variance (12.1\%). In the same region, Conceição et al. (2009) identified a QTL involved with Al tolerance in maize associated with the marker Umc1318. Some authors reported that this region is near the QTL mapped by Sibov et al. (1999) at bin 10.03 called Alm1. Krill et al. (2010) likewise found a QTL in bin 10.04 of this chromosome. The authors attributed this QTL to the gene ZmALMT2 (Zea mays aluminum-activated malate transporter 2). The proteins of the ALMT2 family are related to Al tolerance in wheat, Arabdopsis, and canola. When activated by toxic Al levels, the proteins code a malate transporter that chelates the element and induces a conformational change, opening the anion channels for later exudation by root cells (Krill et al. 2010). Recently, Matiello et al. (2012) confirmed through use of microarray technology that this region could anchor Al tolerance genes. The Zm.5637.4.A1 gene, a marker located inside the Alm1 QTL (Sibov et al. 1999), codes for precursor 8 from the protein xyloglucan endo-transglycosylase, which interacts with new xyloglucans synthetized in the cell walls that contribute to root growth. The authors emphasize that the constitutive expression of this gene in tolerant genotypes indicates that the continuous process of cell wall renovation may be relevant to Al tolerance in maize.

Maron et al. (2010) mapped a second MATE family member (called ZmMATE2), showing it to be the major cause of phenotypic variance (16\%) for maize Al tolerance. This gene was mapped between markers Bnlg105 and Umc1056, located at bins 5.02 and 5.03, respectively. However, this study shows that this gene is different from ZmMATE1 and other MATEs involved in Al tolerance in several aspects. In addition to not being regulated by the presence of Al, ZmMATE2 does not share the same identity with the MATEs in amino acid sequences that are involved in citrate transport, so it could be involved with a different tolerance mechanism. In this study, the identification of a possible QTL mapped at only a $3.6 \mathrm{cM}$ distance from the ZmMATE2 marker explains the $7.8 \%$ of phenotypic variance of Al tolerance. Despite the lower contribution of this QTL compared to the one described by Maron et al. (2010), it can be inferred that this region is associated with Al tolerance in maize. In contrast, the study presented by Guimarães et al. (2014) revealed that the Al tolerance related to the QTL mapped in the qALT5 region is not controlled by the ZmMATE2 gene. Although they did not observe differential gene expression between tolerant and sensitive lines, some authors have confirmed that the amino acid sequences in both genotypes for the ZmMATE2 gene are identical. Additionally, the same study identified qALT5 as outside of the confidence interval, but next to the QTL peak known as the Nrat1 rice homologue gene. The OsNrat1 gene encodes a specific Al transporter (Nramp) involved with Al intracellular absorption in rice. Some studies demonstrated that ZmNrat1 expression is quickly induced in tolerant maize genotypes, while in sensitive genotypes, it takes more time. Despite the absence of significance of the QTL that contain ZmNrat1, homology with Nrat1 of rice 
and differential expression in tolerant and sensitive genotypes justify greater investigation of involvement of this gene in the internal detoxification mechanism of $A 1$ in maize.

The QTL mapped on chromosome 2 at the $34.0 \mathrm{cM}$ position represented the smallest contribution to phenotypic variance, with only $2.1 \%$ of $\mathrm{Al}$ tolerance in the population evaluated. This region was mapped $18.8 \mathrm{cM}$ from the SSR marker Bnlg125, with a recognized position at bin 2.02. On the same chromosome, Ninamango-Cárdenas et al. (2003) identified a QTL on bin 2.06, which explained 10.9\% of phenotypic variance. Matiello et al. (2012) state that there are currently no known functions for this genomic region.

Two other QTL were mapped on chromosome 4 at the $125.0 \mathrm{cM}$ and $197.0 \mathrm{cM}$ positions. Despite being at a $72 \mathrm{cM}$ distance from each other, the SRR reference marker Bnlg1337, positioned at bin 4.11, is the nearest reference to both QTLs. There is no other scientific publication that relates Al tolerance to QTL associated with this region at chromosome 4. However, Conceição et al. (2009) and Krill et al. (2010) have identified significant QTL at bin 4.03. Krill et al. (2010) associated this QTL with Al tolerance to the SAHH (S-adenosyl-L-homocysteine) gene, which actives a SAM (S-adenosyl methionine) inhibitor that mediates the methylation of any organism. Studies published by Eticha et al. (2005) and Yang et al. (2008) have described correlation between pectin methylation from the cell wall with tolerance and exclusion of $\mathrm{Al}$ in maize and rice roots, respectively. This confirms the possibility of $S A H H$ being involved in expression of Al tolerance through cell wall modification.

The last two QTL mapped in this study, at chromosomes 7 and 9, showed lower contribution to phenotypic variance in Al tolerance (2.7\% and 3.1\%, respectively). The QTL at chromosome 7 was mapped only $2.1 \mathrm{cM}$ from the SSR marker Umc1799, located in bin 7.06. The QTL at chromosome 9 was mapped at $10.8 \mathrm{cM}$ from Umc2084 (SSR marker; bin 9.01). This is the first study that has mapped QTL for Al tolerance at these chromosomes/positions. According to the consensus loci of the microsatellite maize map from the Maize Genetics and Genomics Data Base - MaizeGDB, there are no references to Al tolerance genes for these two genomic regions.

The large number of transgressive segregants observed in the $F_{2: 3}$ mapping population allows inferences to be made about a combination of complementary positive alleles from both tolerant and sensitive parents, which results in higher Al tolerance. These transgressive individuals can be used to generate superior inbred lines, with Al tolerance. In summary, three maize genomic regions were confirmed in the present study, coinciding with most QTL mapping studies involving Al tolerance. In addition to showing their contribution to most phenotypic variance in tolerance, these regions were associated with reference genes in the maize genome associated with Al tolerance mechanisms. Chromosome 6 exhibits the main and best-described genomic region (bin 6.00), followed by chromosome 8 (bins 8.04 to 8.07 ) and chromosome 10 (bins 10.01 and 10.04). The high number of QTL mapped in this segregating population confirms the quantitative inheritance pattern for Al tolerance in maize of tropical origin.

\section{REFERENCES}

Basten CJ, Weir BS and Zeng BZ (2005) QTL cartographer version 1.17 (software). North Carolina State University, Raleig, 190p.

Boscolo PR, Menossi M and Jorge RA (2003) Aluminum-induced oxidative stress in maize. Phytochemistry 62: 181-189.

Clay NK and Nelson T (2005) Arabidopsis thickvein mutation affects vein thickness and organ vascularization and resides in a provascular cellspecific spermine synthase involved in vein definition and in polar auxin transport. Plant Physiology 138: 767-777.

Coelho CJ, Molin D, Wood Joris HA, Caires EF, Gardingo JR and Matiello RR (2015) Selection of maize hybrids for tolerance to aluminum in minimal solution. Genetics and Molecular Research 14: 134-144.

Conceição LDHCS, Tessele C and Barbosa Neto JF (2009) Diallel analysis and mapping of aluminum tolerance in corn inbred lines. Maydica 54: $55-61$
Creste S, Tulmann Neto A and Figueira A (2001) Detection of single sequence repeat polymorphisms in denaturing polyacrylamide sequencing gels by silver staining. Plant Molecular Biology Report 19: 299-306.

Doncheva S, Amenós M, Poschenrieder C and Barceló J (2005) Root cell patterning: a primary target for aluminium toxicity in maize. Journal of Experimental Botany 56: 1213-1220.

Eticha D, Stass A and Horst WJ (2005) Cell-wall pectin and its degree of methylation in the maize root-apex: significance for genotypic differences in aluminum resistance. Plant Cell \& Environment 28: $1410-1420$

Guimarães CT, Simões CC, Pastina MM, Maron LG, Magalhães JV and Vasconcellos RCC (2014) Genetic dissection of Al tolerance QTLs in the maize genome by high density SNP scan. BMC Genomics 15: 1-14.

Haldane's Mapping Function (2008) In: Rédei, GP. Encyclopedia of Genetics, Genomics, Proteomics and Informatics. $3^{\text {rd }}$ ed. Springer, 


\section{CJ Coelho et al.}

Dordrecht, p. 365-367.

Hoisington D, Khairallah M, Gonzälez de Leon D (1994) Laboratory protocols: CIMMYT applied molecular genetics laboratory. $2^{\text {nd }} \mathrm{ed}$. México: CIMMYT, 51p.

Krill AM, Kirst M, Kochian LV, Buckler ES and Hoekenga OA (2010) Association and linkage analysis of aluminum tolerance genes in maize. PLoS ONE 5: 1-11.

Lander ES, Green P, Abrahamson J, Barlow A, Daly MJ, Lincoln SE and Newburg L (1987) Mapmaker: an interactive computer package for constructing primary genetic linkage maps of experimental and natural populations. Genomics 1: 174-181.

Maron LG, Piñeros MA, Guimarães CT, Magalhães JV, Pleiman JK, Mao C, Shaff J, Belicuas SN and Kochian LV (2010) Two functionally distinct members of the MATE (multi-drug and toxic compound extrusion) family of transporters potentially underlie two major aluminum tolerance QTLs in maize. Plant Journal 61: 728-740.

Matiello L, Silva FR and Menossi M (2012) Linking microarray data to QTLs highlights new genes related to Al tolerance in maize. Plant Science 191: 8-15.

Ninamango-Cárdenas FE, Guimarães $\mathrm{CT}$, Martins PR, Parentoni SN, Carneiro NP, Lopes MA, Moro JR and Paiva E (2003) Mapping QTLs for aluminum tolerance in maize. Euphytica 130: 223-232.

Ogliari JB, Boscariol RL and Camargo LES (2000) Optimization of PCR amplification maize microsatellites loci. Genetics and Molecular Biology 23: 395-398.

Orzaez D, de Jong AJ and Woltering EJ (2001) A tomato homologue of the human protein PIRIN is induced during programmed cell death. Plant Molecular Biology 46: 59-468.

Pandey S, León LAN, Friese DK and Waddington SR (2007) Breeding maize for tolerance to soil acidity. Plant Breeding 28: 59-100.

Piñeros MA, Magalhães JV, Carvalho Alves VM and Kochian LV (2002) The physiology and biophysics of an aluminum tolerance mechanism based on root citrate exudation in maize. Plant Physiology 129: 1194-1206.

Piñeros MA, Shaff JE, Manslank HS, Carvalho Alves VM and Kochian LV (2005) Aluminum resistance in maize cannot be solely explained by root organic acid exudation: A comparative physiological study. Plant Physiology 137: 231-241.

R Core Team (2017) R: A language and environment for statistical computing. R Foundation for Statistical Computing, Vienna, Austria. URL https://www.R-project.org/.

Senior ML, Chin E, Austin D, Lee M and Smith S (1996) Mapping simple sequence repeats in maize. Maize Newsletter 70: 50-54

Sibov ST, Gaspar M, Silva MJ, Ottoboni LMM, Arruda P and Souza AP (1999) Two genes control aluminum tolerance in maize: genetic and molecular mapping analyses. Genome 42: 475-482.

Vos P, Hogers R, Bleeker M, Reijans M, van de Lee T and Hornes M (1995) AFLP: a new technique for DNA fingerprinting. Nucleic Acids Research 23: 4407-4414.

Yang JL, Li YY, Zhang YJ, Zhang SS, Wu YR, Wu P and Zheng SJ (2008) Cell wall polysaccharides are specifically involved in the exclusion of aluminum from the rice root apex. Plant Physiology 146: 602-611. 